\title{
Qual o nível de competição da mucuna-preta com a cultura do amendoim?
}

Submetido - 27 jul. $2020 \quad$ Aprovado - 10 set. $2020 \quad$ Publicado - 14 out. 2020

\begin{abstract}
Ebson Silva (iD
Graduando em Agronomia - Faculdade de Ciências Agrárias e Veterinárias/Universidade Estadual Paulista FCAV/UNESP, e-mail: ebson.silva@etec.sp.gov.br.
\end{abstract}

Ana Eliza Piazentine (iD

Mestranda em Produção Vegetal - Faculdade de Ciências Agrárias e Veterinárias/Universidade Estadual Paulista FCAV/UNESP, e-mail: elizapiazentine@hotmail.com.

\section{Leandro Aparecido Chiconi (iD}

Graduando em Agronomia - Faculdade de Ciências Agrárias e Veterinárias/Universidade Estadual Paulista FCAV/UNESP, e-mail: leandroapchiconi@hotmail.com.

Maira Rosene da Costa ${ }^{(D)}$

Graduanda em Ciências Biológicas - Faculdade São Luís, e-mail: mairaxmc@gmail.com.

\section{Willians César Carrega iD}

Doutor em Produção Vegetal - Faculdade de Ciências Agrárias e Veterinárias/Universidade Estadual Paulista FCAV/UNESP, e-mail: willianscesar@hotmail.com.

Pedro Luís da Costa Aguiar Alves (iD

Doutor em Biologia Vegetal - Faculdade de Ciências Agrárias e Veterinárias/Universidade Estadual Paulista FCAV/UNESP, e-mail: plalves@fcav.unesp.br.

\section{RESUMO}

A interferência de plantas daninhas em convivência com o amendoim afeta o crescimento e desenvolvimento das plantas, podendo causar perdas superiores a $80 \%$ na produtividade da cultura. Dentre as principais espécies daninhas problemáticas nas áreas de produção de amendoim, tem-se observado aumento significativo das plantas trepadeiras, principalmente, da mucuna e cordas-de-viola. Diante disso, objetivou-se com este trabalho avaliar o impacto da competição da mucuna-preta no desenvolvimento das plantas e na produtividade do amendoim. $O$ experimento foi conduzido em campo no delineamento em blocos casualizados, com 5 repetições. Os tratamentos foram constituídos por seis densidades de mucuna-preta (0, $1,2,3,4$ e 5 plantas $\left(m^{2}\right)$ em competição com a cultivar de amendoim IAC OL3. Durante os estágios reprodutivos $R 1$ a $R 7$, foram realizadas avaliações da radiação fotossinteticamente ativa interceptada pelas plantas daninhas e pela cultura do amendoim. Ao final do período experimental (130 DAS), foi realizada a colheita manual do amendoim e da mucuna-preta. Em seguida, foi determinada a massa seca da parte aérea da mucuna-preta e do amendoim. Determinou-se, também, o número e massa das vagens e sementes do amendoim. Os dados obtidos foram submetidos a análise de variância pelo teste $F$ e as médias comparadas pelo teste de Tukey ao nível de $5 \%$ de probabilidade. Com base nos resultados obtidos, verificou-se que a massa vegetativa da mucuna-preta, independente da densidade de plantas, proporcionou sombreamento no amendoim, reduzindo, em média, $90 \%$ a interceptação da radiação fotossinteticamente ativa pela cultura, $70 \%$ a massa vegetativa do amendoim e $76 \%$ a produtividade de vagens e sementes do amendoim. Diante disso, conclui-se que a mucunapreta é uma planta bastante agressiva em competição com a cultura do amendoim e causa sérios prejuízos na densidade de uma ou mais plantas $/ \mathrm{m}^{2}$.

Palavras-chave: Arachis hypogaea L.; Mucuna aterrima; Produtividade; Radiação fotossinteticamente ativa; plantas daninhas. 


\title{
What is the level of competition between the black mucuna and the peanut culture?
}

\begin{abstract}
The interference of weeds in coexistence with peanuts affects the growth and development of plants, which can cause losses of more than $80 \%$ in crop productivity. Among the main problematic weeds in the peanut production areas, there has been a significant increase in climbing plants, mainly mucuna and viola cords. Therefore, the objective of this work was to evaluate the impact of the competition of the black mucuna in the development of the plants and in the productivity of the peanut. The experiment was conducted in the field in a randomized block design, with 5 replications. The treatments consisted of six densities of black mucuna $(0,1,2,3,4$ and 5 plants / m2) in competition with the peanut cultivar IAC OL3. During the reproductive stages $R 1$ to $R 7$, evaluations of photosynthetically active radiation intercepted by weeds and peanut culture were carried out. At the end of the experimental period (130 DAS), peanuts and black mucuna were harvested manually. Then, the dry mass of the aerial part of the mucuna-negra and peanut was determined. The number and mass of the peanut pods and seeds were also determined. The data obtained were subjected to analysis of variance by the $F$ test and the means compared by the Tukey test at the level of $5 \%$ probability. Based on the results obtained, it was found that the vegetative mass of the black mucuna, regardless of plant density, provided shading in the peanuts, reducing, on average, $90 \%$ the interception of photosynthetically active radiation by the crop, $70 \%$ the vegetative mass peanut and $76 \%$ the productivity of peanut pods and seeds. Therefore, it is concluded that the black mucuna is a very aggressive plant in competition with the peanut culture and causes serious losses in the density of one or more plants $/ \mathrm{m}^{2}$.
\end{abstract}

Keywords: Arachis hypogaea L.; Mucuna aterrima; Productivity; Photosynthetically active radiation; Weeds.

\section{¿Cuál es el nivel de competencia entre la mucuna negra y la cultura del maní?}

\section{RESUMEN}

La interferencia de las malas hierbas en la convivencia con el maní afecta el crecimiento y desarrollo de las plantas, que pueden provocar pérdidas de más del $80 \%$ en la productividad de los cultivos. Entre las principales malezas problemáticas en las zonas productoras de maní, se ha observado un aumento significativo de plantas trepadoras, principalmente cuerdas de mucuna y viola. Por tanto, el objetivo de este trabajo fue evaluar el impacto de la competencia de la mucuna negra en el desarrollo de las plantas y en la productividad del maní. El experimento se realizó en campo en un diseño de bloques al azar, con 5 repeticiones. Los tratamientos consistieron en seis densidades de mucuna negra (0, 1, 2, 3, 4 y 5 plantas / m2) en competencia con el cultivar de maní IAC OL3. Durante las etapas reproductivas $R 1$ a $R 7$, se realizaron evaluaciones de radiación fotosintéticamente activa interceptada por malezas $y$ cultivo de maní. Al final del período experimental (130 DAS), el maní y la mucuna negra se recolectaron manualmente. Luego, se determinó la masa seca de la parte aérea de la mucunanegra y maní. También se determinó el número y la masa de las vainas y semillas de maní. Los datos obtenidos se sometieron a análisis de varianza mediante la prueba $F$ y las medias se compararon mediante la prueba de Tukey al nivel de $5 \%$ de probabilidad. Con base en los resultados obtenidos, se encontró que la masa vegetativa de la mucuna negra, independientemente de la densidad vegetal, proporcionó sombreado en el maní, reduciendo, en promedio, en un $90 \%$ la interceptación de la radiación fotosintéticamente activa por parte del cultivo, en un $70 \%$ la masa vegetativa maní y $76 \%$ la productividad de las vainas y semillas de maní. Por tanto, se concluye que la mucuna negra es una planta muy agresiva en competencia con el cultivo del maní y provoca graves pérdidas en la densidad de una o más plantas / $\mathbf{m}^{2}$.

Palabras clave: Arachis hypogaea L; Mucuna aterrima; Productividad, Radiación fotosintéticamente activa; Malezas. 


\section{Introdução}

O amendoim (Arachis hypogaea L.) é cultura de importância econômica amplamente comercializada em todo o mundo (DING et al. 2017). No Brasil, a produção está concentrada no Estado de São Paulo, que detém $93,6 \%$ da produção total (CONAB, 2020).

Nos últimos anos, a cultura do amendoim no Brasil tem apresentado crescimento significativo em área de semeadura e em produtividade, devido principalmente a inserção da cultura em áreas em reforma de canaviais. Entretanto, as culturas estão sujeitas a fatores que podem causar interferências negativas na produtividade. Dentre eles, a competição das plantas daninhas com o amendoim pode causar perdas de até $80 \%$ (NEPOMUCENO et al., 2007).

As plantas daninhas competem com as culturas agrícolas principalmente por água, luz, nutrientes e espaço (PITELLI, 1987). Espécies trepadeiras, como as mucuna-preta e cordas de viola, são plantas que além de competir por água e nutrientes, tem forte potencial competitivo por luz e espaço, já que essas plantas se entrelaçam nas culturas, causando o sombreamento e ocupando maior espaço, não permitindo a expansão (i.e. desenvolvimento) da cultura.

De acordo com Caron et al. (2003), a absorção de luz é de fundamental importância para a realização dos principais processos fisiológicos, dentre eles, a fotossíntese. Como plantas de mucuna-preta se entrelaçam nas culturas, é necessário determinar qual o impacto que essas plantas podem ter sob a capacidade de interceptação de radiação fotossinteticamente ativa pelo amendoim.

Diante disso, objetivou-se com este trabalho avaliar a interceptação da radiação solar do amendoim em competição com densidades de mucunapreta, visando determinar o impacto na produtividade da cultura. 


\section{Material e métodos}

O experimento foi desenvolvido em condições semi-controladas no Laboratório de Plantas Daninhas (LAPDA) na Faculdade de Ciências Agrárias e Veterinárias da Universidade Estadual Paulista (FCAV/UNESP), Campus de Jaboticabal.

O delineamento experimental foi em blocos casualizados, com 6 densidades de mucuna-preta $\left(0,1,2,3,4\right.$ e 5 plantas $\left./ \mathrm{m}^{2}\right)$. A cultivar de amendoim utilizada foi a IAC OL3, que possui ciclo curto (cerca de 125 e 130 dias), teor de ácido oleico variando entre (70 e $80 \%)$ e produtividade média de $4.500 \mathrm{~kg} / \mathrm{ha}$.

As sementes foram previamente tratadas com 0 inseticida tiametoxan (Cruiser® - $200 \mathrm{~mL}$ do p.c./100 kg de sementes) e o fungicida carboxina + tiram (Vitavax®-Thiram $200 \mathrm{SC}-350 \mathrm{~mL}$ do p.c. $/ 100 \mathrm{~kg}$ de sementes). Em seguida, a semeadura foi realizada em caixas de amianto $(74 \mathrm{~cm} \times 74 \mathrm{~cm} \times 25 \mathrm{~cm})$, com capacidade de $87 \mathrm{~L}$.

Em cada caixa foram semeadas 30 sementes de amendoim/metro, utilizando uma mistura de solo (Latossolo Vermelho Escuro e Areia de Rio) na proporção 2:1. Após a emergência do amendoim, foi realizado o desbaste deixando 12 sementes/metro. Após 15 dias da semeadura do amendoim, foi realizado o plantio da mucuna-preta de acordo com as densidades $(0,1$, 2, 3, 4 e 5 plantas $/ \mathrm{m}^{2}$ ).

Foram realizadas aplicações de inseticidas e fungicidas a cada sete dias, visando o controle fitossanitário, e realizou-se a irrigação sempre que necessária, mantendo o solo a $80 \%$ da capacidade de campo. Aos 47, 59, 72, 80, 95 e 111 dias após a semeadura (DAS), foi realizada a determinação da interceptação da radiação fotossinteticamente ativa (RFA), utilizando ceptômetro AccuPAR LP- 80 (Decagon devices, Inc., Pullman, EUA), que é uma sonda com 80 sensores quânticos de radiação PAR (400 a 700 nm).

As avaliações aos 47 e 59 DAS foram realizadas determinando a RFA acima e abaixo do dossel do amendoim, visto que, a mucuna-preta 
ainda não apresentava tamanho suficiente para interceptar a radiação solar da cultura. Após os 59 DAS, as determinações da RFA levaram em consideração o sombreamento da mucuna-preta no amendoim e, por isso, foram realizadas avaliações acima, no meio e abaixo dossel das plantas.

Ao final do experimento (130 DAS), foi realizada a colheita manual do amendoim e da mucuna-preta e as plantas foram acondicionadas em sacos de papel, devidamente identificados e colocadas para secar em estufa a $65^{\circ} \mathrm{C}$ por 72 horas para determinação da massa seca da parte aérea das plantas. Além disso, após a colheita, foi determinado o número e massa das vagens.

Os dados obtidos foram submetidos a análise de variância pelo teste $F$, com as médias comparadas pelo teste de Tukey ao nível de $5 \%$ de probabilidade. Quando significativo, as médias foram submetidas à análise de regressão.

\section{Resultados e discussão}

Ao longo do desenvolvimento das plantas ( $R 1$ a $R 6$ ), a presença da mucuna-preta afetou significativamente a interceptação da RFA (dados não apresentados). No Início da maturação (estágio R7), observou-se que todas as densidades de mucuna-preta reduziram a interceptação da RFA pelo amendoim. Essas reduções foram de $83 \%$ (1 planta/ $\left./ \mathrm{m}^{2}\right), 86 \%$ (2 plantas $\left./ \mathrm{m}^{2}\right)$, 92\% (3 plantas $\left./ \mathrm{m}^{2}\right), 93 \%$ (4 plantas $/ \mathrm{m}^{2}$ ) e de $92 \%$ (5 plantas $\left./ \mathrm{m}^{2}\right)$.

A RFA efetivamente interceptada pelas plantas de amendoim foi de $9 \%, 8 \%, 4 \%, 2 \%$ e $5 \%$, nas densidades de $1,2,3,4$ e 5 plantas $/ \mathrm{m}^{2}$, demonstrando, assim, alto potencial competitivo por essas plantas pela RFA. Para as plantas de amendoim sem competição com a mucuna-preta, verificou-se que a interceptação de RFA foi de $82,89 \%$ (Figura 1A e 1B).

Sem levar em consideração as densidades (média geral), a eficiência na interceptação da RFA pelo amendoim sem competição com a mucuna-preta foi de $82,89 \%$ (Figura $2 \mathrm{~A}$ ). No entanto, quando as plantas 
estavam sob competição com a mucuna-preta, o amendoim conseguiu interceptar, em média, apenas 6,16\% (Figura 2B).

A

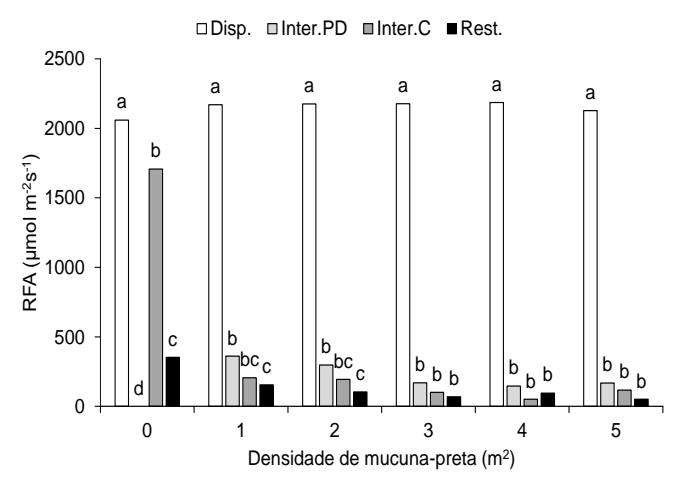

B

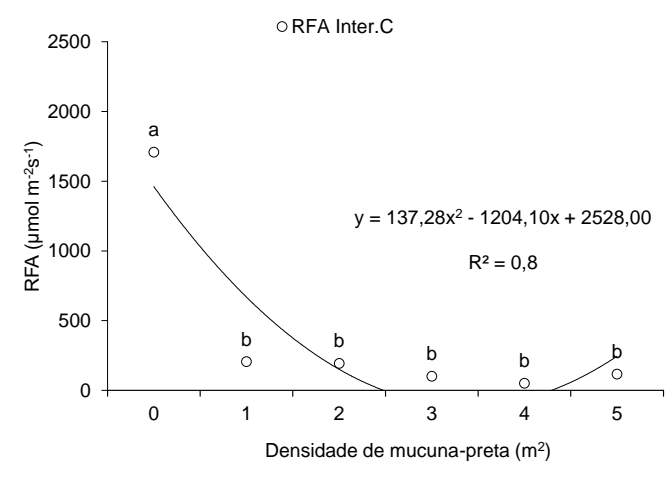

Figura 1. Competição da mucuna-preta ( $\mathrm{A}$ e B) na interceptação de radiação fotossinteticamente ativa (RFA) durante o início da maturação (estágio R7) do amendoim. A, RFA para cada densidade; acima do dossel (disponibilidade total de RFA), meio do dossel (RFA interceptada (PD) pelas plantas daninhas), abaixo do dossel (RFA restante) e relação meio e abaixo do dossel (RFA interceptada(C) pela cultura). B, RFA interceptada entre as densidades.

A

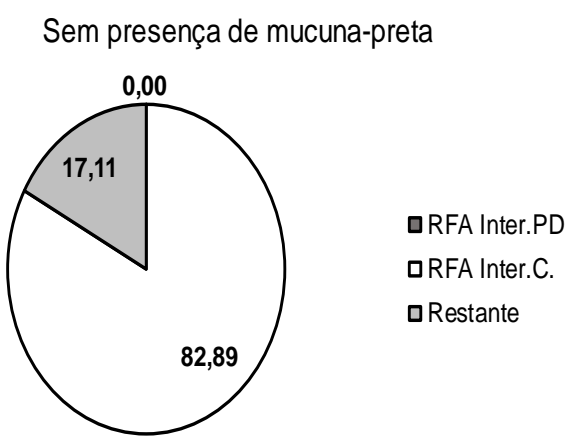

B

Com presença de mucuna-preta

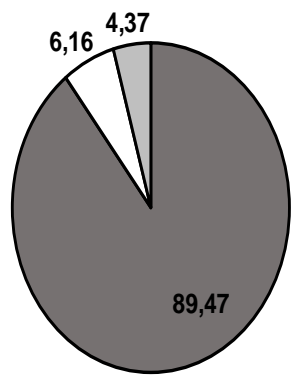

QRFA Inter.PD

口RFA Inter.C.

口Restante

Figura 2. Média geral da interferência da mucuna-preta na interceptação da radiação fotossinteticamente ativa em competição com a cultura do amendoim ao final do ciclo da cultura.

Para a massa seca da parte aérea acumulada pela mucuna-preta, verificou-se que partir de 1 planta de mucuna-preta $/ \mathrm{m}^{2}$ ocorreu aumento significativo na massa vegetativa, chegando a 800 g.m $\mathrm{m}^{-1}$ na densidade de 5 plantas $/ \mathrm{m}^{2}$ (Figura 3A). Essa quantidade de massa vegetativa acumulada pela planta daninha, proporcionou forte sombreamento no amendoim, interceptando $\cong 90 \%$ da RFA disponível para a cultura (Figura 2 B), levando 
à redução no crescimento $\mathrm{e}$ desenvolvimento das plantas, e consequentemente, no acúmulo de massa vegetativa pela cultivar IAC OL3. As reduções na massa do amendoim foram observadas a partir da densidade de 1 planta $/ \mathrm{m}^{2}$, chegando a reduzir $80 \%$ quando o amendoim estava sob competição na densidade de 5 plantas de mucuna-preta $/ \mathrm{m}^{2}$ (Figura 3B).

A

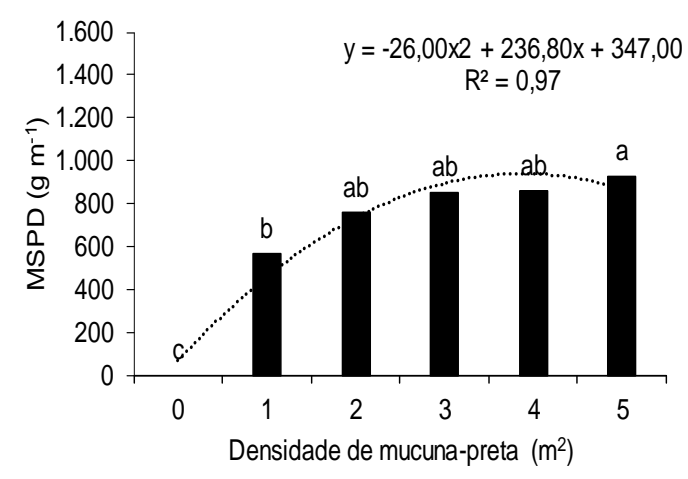

C

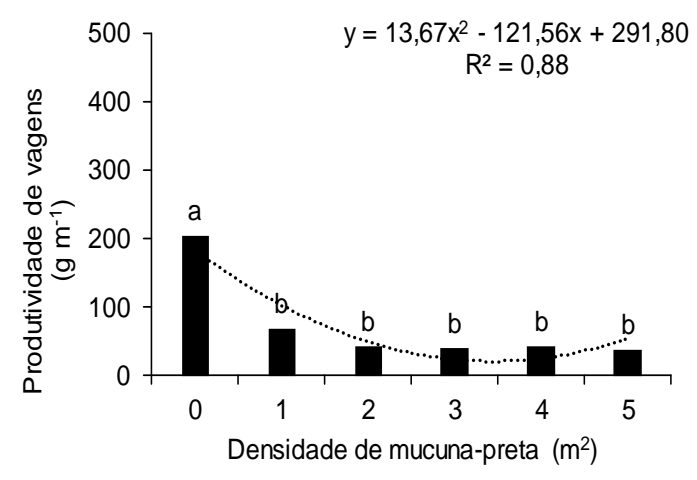

B

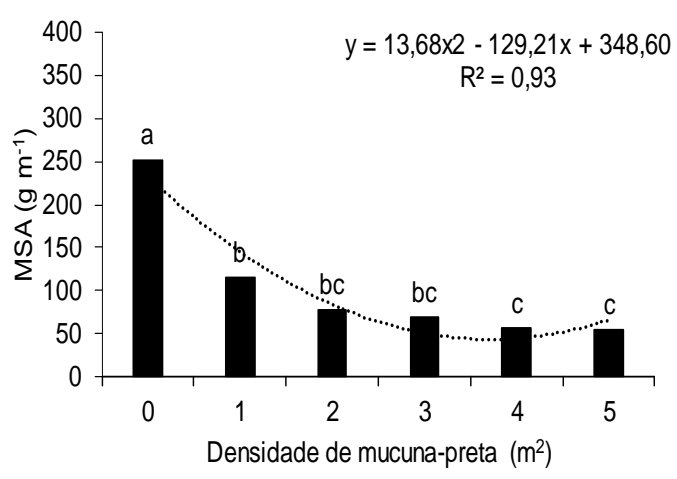

D

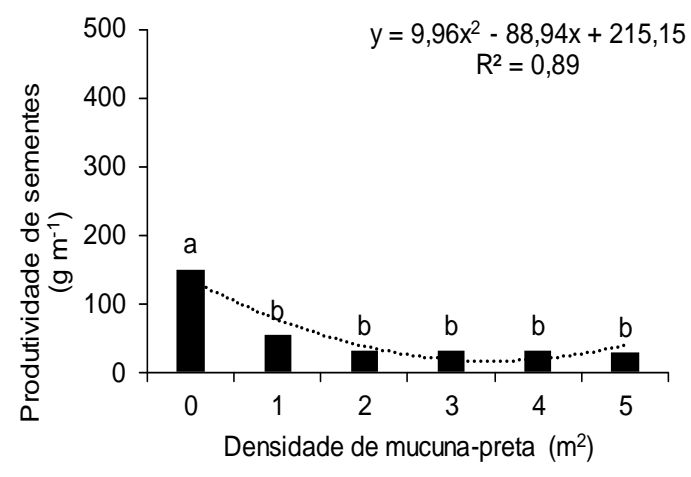

Figura 3. Massa seca da parte aérea da mucuna-preta (A), do amendoim em convivência com a mucuna-preta (B) e produtividade de vagens (C) e sementes (D) de amendoim em convivência.

A produtividade de vagens e de sementes do amendoim (Figura $3 \mathrm{C}$ e 3D), foi reduzida significativamente pela competição com a mucuna-preta, independente da densidade de plantas. Essa redução foi, em média, de $76 \%$, quando comparada com as plantas de amendoim sem competição com a mucuna-preta. Este resultado é reflexo da baixa RFA interceptada pelo amendoim ( $\cong 6 \%$, Figura $2 \mathrm{~B}$ ), a qual refletiu no menor desenvolvimento das plantas e que, consequentemente, acarretou em menor produtividade de 
vagens e sementes. Nakagawa et al. (1994) verificaram que o aumento populacional de plantas diminui a produtividade das vagens.

\section{Conclusões}

A mucuna-preta é uma planta bastante agressiva em convivência com a cultura do amendoim, sendo prejudicial na densidade de uma ou mais plantas $/ \mathrm{m}^{2}$, quando, em média, reduz $\cong 90 \%$ da interceptação da radiação fotossinteticamente ativa, $770 \%$ da massa vegetativa e $\cong 76 \%$ da produtividade de vagens e sementes do amendoim. 


\section{Referências}

CARON, B. O.; MEDEIROS, S. L. P.; MANFRON, P. A.; SCHMIDT, D.; POMMER, S. F.; BIANCHI, C. Influência da temperatura do ar e radiação solar no acúmulo de fitomassa da alface. Revista Brasileira de Agrometeorologia, v. 11, n. 2, p. 275-283, 2003.

CONAB. COMPANHIA NACIONAL DE ABASTECIMENTO. Acompanhamento da Safra Brasileira de Grãos 2019/2020. Disponível em: http:// www.conab.gov.br/info-agro/safras/graos/. Acesso em: 20 mar. 2020.

DING, H.; ZHANG, Z.; KANG, T.; DAI, L.; CI, D.; QIN, F.; SONG, W. (2017). Rooting traits of peanut genotypes differing in drought tolerance under drought stress. International Journal of Plant Production, v. 11, p. 349360, 2017. https://doi.org/10.22069ijpp.2017.3544

NAKAGAWA, J.; LASCA, D.H.C.; NEVES, J.PS.; NEVES, G.S.; SANCHES, S. V.; BARBOSA, V.; SILVA, M.N.; ROSSETTO, C.A.V. Efeito da densidade de semeadura na produção do amendoim. Pesquisa Agropecuária Brasileira, Brasília, v. 29, n. 10, p. 1547-555, 1994.

NEPOMUCENO, M. P.; ALVES, P.L.C.A.; DIAS, T.C.S.; CARDOZO, N.P.; PAVANI, M.C.M.D. Efeito da época de semeadura nas relações de interferência entre uma comunidade infestante e a cultura do amendoim. Planta Daninha, v. 25, n. 3, p. 481-488, 2007. Planta Daninha. https://doi.org/10.1590/S0100-83582007000300007

PITELLI, Robinson Antonio. Competição e controle de plantas daninhas em áreas agrícolas. Série TÉCNICA IPEF, v.4, n.12, p.1-24, 1987. 